\title{
Hierarchical structure formation in layered superconducting systems with multi-scale inter-vortex interactions
}

\author{
Christopher N. Varney ${ }^{1,2}$, Karl A. H. Sellin ${ }^{3}$, Qing-Ze Wang ${ }^{1,4}$, \\ Hans Fangohr ${ }^{5}$, and Egor Babaev ${ }^{1,3}$ \\ ${ }^{1}$ Department of Physics, University of Massachusetts, Amherst, Massachusetts 01003, \\ USA \\ ${ }^{2}$ Department of Physics, University of West Florida, Pensacola, Florida 32514, USA \\ ${ }^{3}$ Department of Theoretical Physics, The Royal Institute of Technology, SE-10691 \\ Stockholm, Sweden \\ ${ }^{4}$ Department of Physics, The Pennsylvania State University, University Park, \\ Pennsylvania 16802, USA \\ ${ }^{5}$ Engineering and the Environment, University of Southampton, Southampton, SO17 \\ 1BJ, UK \\ E-mail: cvarney@uwf .edu
}

\begin{abstract}
We demonstrate formation of hierarchical structures in two-dimensional systems with multiple length scales in the inter-particle interaction. These include states such as clusters of clusters, concentric rings, clusters inside a ring, and stripes in a cluster. We propose to realize such systems in vortex matter (where a vortex is mapped onto a particle with multi-scale interactions) in layered superconducting systems with varying inter-layer thicknesses and different layer materials.

PACS numbers: $74.25 . \mathrm{Uv}, 74.25 . \mathrm{Dw}, 74.45 .+\mathrm{c}, 61.46 . \mathrm{Bc}$
\end{abstract}

\section{Introduction}

Condensed matter physics has long been concerned with explaining phenomena that result from competing interactions, covering a wide variety of topics from soft condensed matter systems to magnetism and ultra-cold atoms (for a recent overview see Refs. [1] and [2]). The richest pattern forming systems are those with several length scales. For example, structure formation in systems with multi-scale interactions is highly relevant in hard condensed matter systems [3-5], nuclear matter [6], and in colloids and other soft condensed matter systems [7,8]. Alternatively, nontrivial patterns can arise from the combination of inter-particle interactions and an external potential [9].

One of the promising systems for non-trivial structure formation are superconducting vortices. Research on the magnetic response of standard type- 2 superconductors traditionally typically deals with structure formation of vortices [10]. Although the 
interaction between vortices in these materials has a simple monotonically repulsive form, the vortex matter exhibits a plethora of interesting phase transitions and structure formation [11]. Moreover, the vortex states, especially in the presence of pinning, are critically important for technological applications of superconductors, where control over vortex matter in many cases amounts to control of dissipation.

Recently, the possibility of more complicated inter-vortex interactions in newly discovered systems has attracted much attention in various contexts: in multicomponent superconductors [1, 12-27], superfluids [28], vortices in dense nuclear matter in neutron stars [29], and quantum Hall systems [5]. Recently a problem which attracted interest was the phase separation in vortex matter with long-range attractive and short-range repulsive inter-vortex interactions. Such forces in multi-component superconductors originate in the regime where there are several superconducting components originating from different bands. This gives rise to two coherence lengths, $\xi_{1}$ and $\xi_{2}$, and the magnetic field penetration length falls between them: $\xi_{1}<\lambda<\xi_{2}[12]$. Such multi-scale long-range attractive, short-range repulsive inter-vortex interaction potentials were derived in a variety of superconducting models: ranging from multicomponent Ginzburg-Landau models with various interband couplings [19] to multiband Eilenberger models [22, 23]. This phenomena, recently referred to as "type-1.5 superconductivity," [13] is the subject of a recent review [30] and has stoked considerable experimental interest in pursuing a realization of such regimes using artificial structures made of alternating layers of type- 1 and type- 2 materials.

Here we propose that superconducting systems can have vortex states with several length scales of repulsive (also in some cases attractive) interactions, where more complicated interaction potentials can be realized. Such inter-vortex forces should arise in layered structures made of combinations of type- 2 and type- 1 superconductors where the magnetic field penetration length $\lambda$ varies in different layers. Since $\lambda$ sets the length scale of the repulsive inter-vortex forces, having a vortex stack piercing several layers, each having its own $\lambda_{i}$ as shown in Figure 1(c) should result in the existence of several repulsive length scales $\lambda_{i}$ in the interaction between such vortices (a vortex stack is kept together due to electromagnetic and inter-layer Josephson coupling). Another way to produce multiple repulsive length scales is to have different insulating layers of different thickness. The thickness determines how much magnetic field lines spread between the layers. The supercurrent on a surface of a superconductor should be determined selfconsistently with the inter-layer field and thus it should result in multiple repulsive length scales. The corresponding interaction length scales should be short range as can be deduced from flux conservation similar to those in Ref. [31]. Also, for quasi-twodimensional systems an additional power-law repulsive interaction is present due to the interaction of stray fields outside the sample [32]. Here we consider the regime where vortex line tension is large and temperature is small so the vortices do not bend. In this limit we can describe the system with an effective two-dimensional model where vortices can be described by their positions in the $x y$-plane. The results, however, are generalizable to the three-dimensional case which we will consider separately. 
(a)

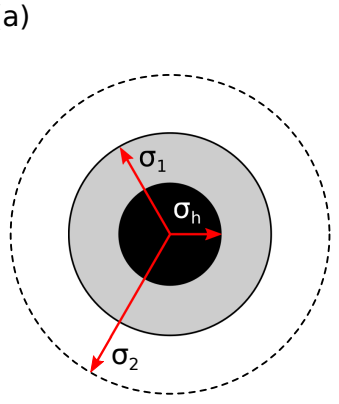

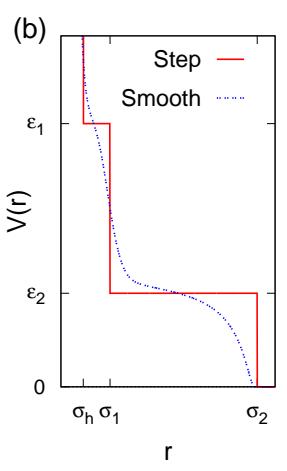

Figure 1: (a) Sketch of a vortex showing interaction length scales. $\sigma_{h}$ is the hardcore radius and $\sigma_{1}\left(\sigma_{2}\right)$ is the inner (outer) soft-core radius. (b) Pair potential $V(r)$ as a function of the inter-particle separation $r$ for a step potential and an analogous smooth potential. (c) Schematic drawing of the field lines of a vortex in a layered superconductor. Shaded (white) regions are superconducting (insulation) of varying thickness. Having layers with different magnetic field penetration lengths allows for multiple repulsive length scales in vortex-vortex interactions. Also by controlling the thickness of the insulating layers one can control the repulsive length scales of the inter-vortex interaction. Thicker insulating layers cause a wider spread of the magnetic field lines resulting in the presence of an additional repulsive length in the inter-vortex interaction scales. Having type-I layers also allows having repulsive scales in the intervortex interaction [30].

In what follows, we use both Monte Carlo (MC) [33] and Langevin dynamics (LD) [34] simulations, which are typically used in studying vortex physics, to demonstrate that vortex systems with competing interactions ranging over multiple length scales exhibit nontrivial hierarchical structure formation, where at short distances the system can form vortex clusters or vortex stripes, which subsequently order themselves into complex patterns at longer length scales. In Section 2, we discuss the ground state vortex configuration of a hard sphere model with several repulsive length scales. Next, we examine the more general case of multi-scale interactions containing regions of repulsion and attraction in Section 3. We summarize the conclusions of this work in Section 4 and conclude with a discussion of the details of the simulation and the potentials studied in Appendix A and Appendix B, respectively.

\section{Purely Repulsive Interactions}

With the physical realization described in Section 1 in mind, let us consider the simplest potential with several length scales, a hard sphere model with multiple shoulders. Note that "core-softened" potentials with a single shoulder have been studied intensively, revealing a myriad of density-modulated ground states [7, 8, 35-37]. In Figures 1(a) and $1(\mathrm{~b})$ we illustrate a particle with an impenetrable hard-core radius $\sigma_{h}$ and two 


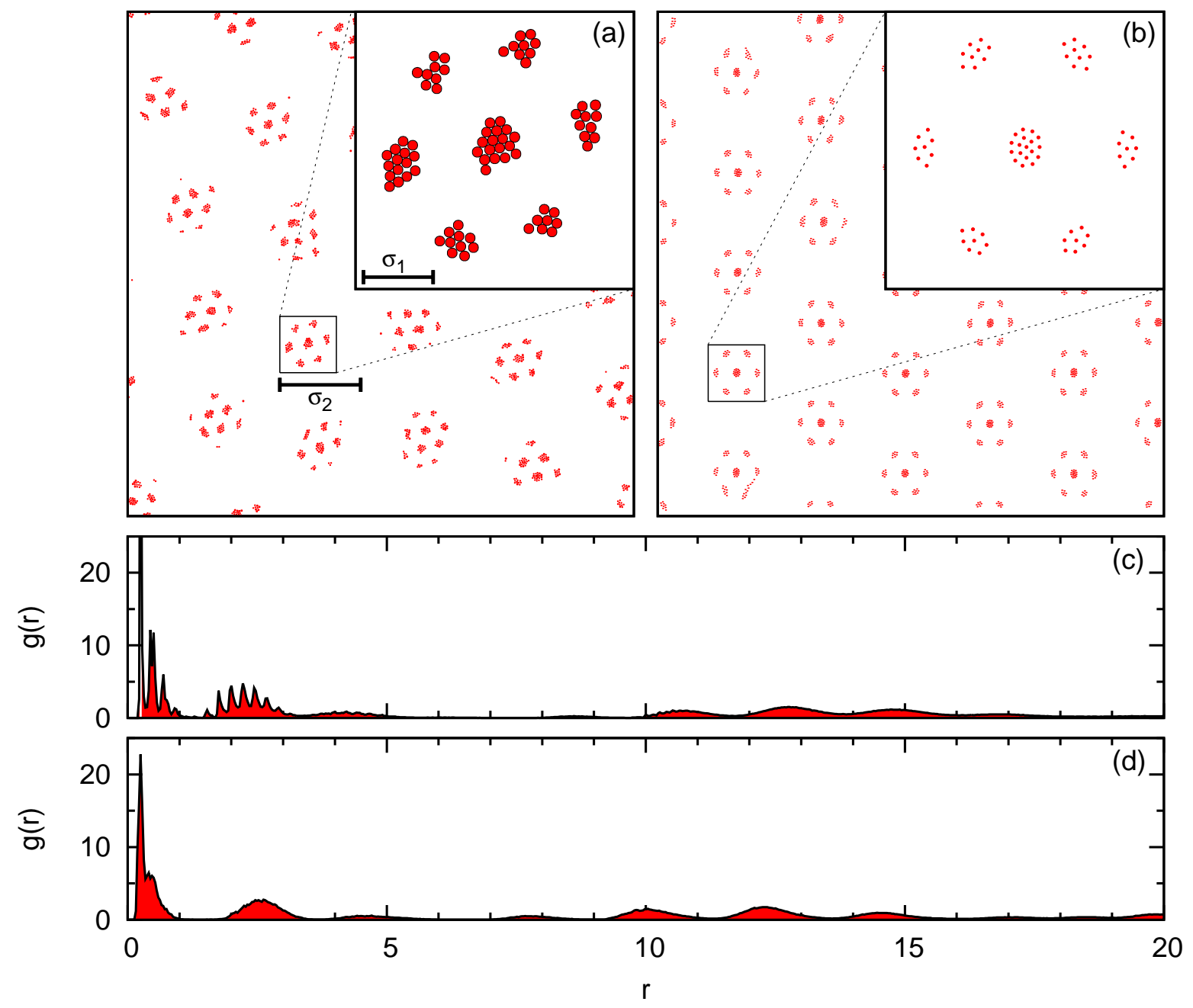

Figure 2: Final particle configuration for (a) three-step potential of Figure 1(b) and (b) smoothed potential with $N_{v}=2000$ and $\rho=0.51$. The corresponding radial distribution functions are plotted versus particle separation (in units of the characteristic simulation length $\lambda$ ) in panels (c) and (d), respectively. The length scales $\sigma_{1}$ and $\sigma_{2}$ are defined in Appendix B.

repulsive shoulders at $r=\sigma_{1}$ and $\sigma_{2}$ with heights $\varepsilon_{1}$ and $\varepsilon_{2}$, respectively.

We show the final configuration from MC simulations of our hard-sphere model in Figure 2(a) for $N_{v}=2000$ particles and density $\rho=0.51$. Here the system forms a hierarchical structure: namely the particles order on three different length scales: (1) the particles form a tightly bound cluster, (2) the clusters are themselves bound into a conglomerate structure (hereafter referred to as a supercluster), and (3) the structures form a lattice. To analyze the underlying structure of this phase, we show the radial distribution function (RDF) $g(r)$ [38] in Figure 2(c). The first feature in $g(r)$ is a very strong peak corresponding to the nearest-neighbor distance inside each cluster. Because the clusters have such a small radius, $g(r)$ shows only a small peak at approximately double the nearest-neighbor distance. The next pronounced peak is the 


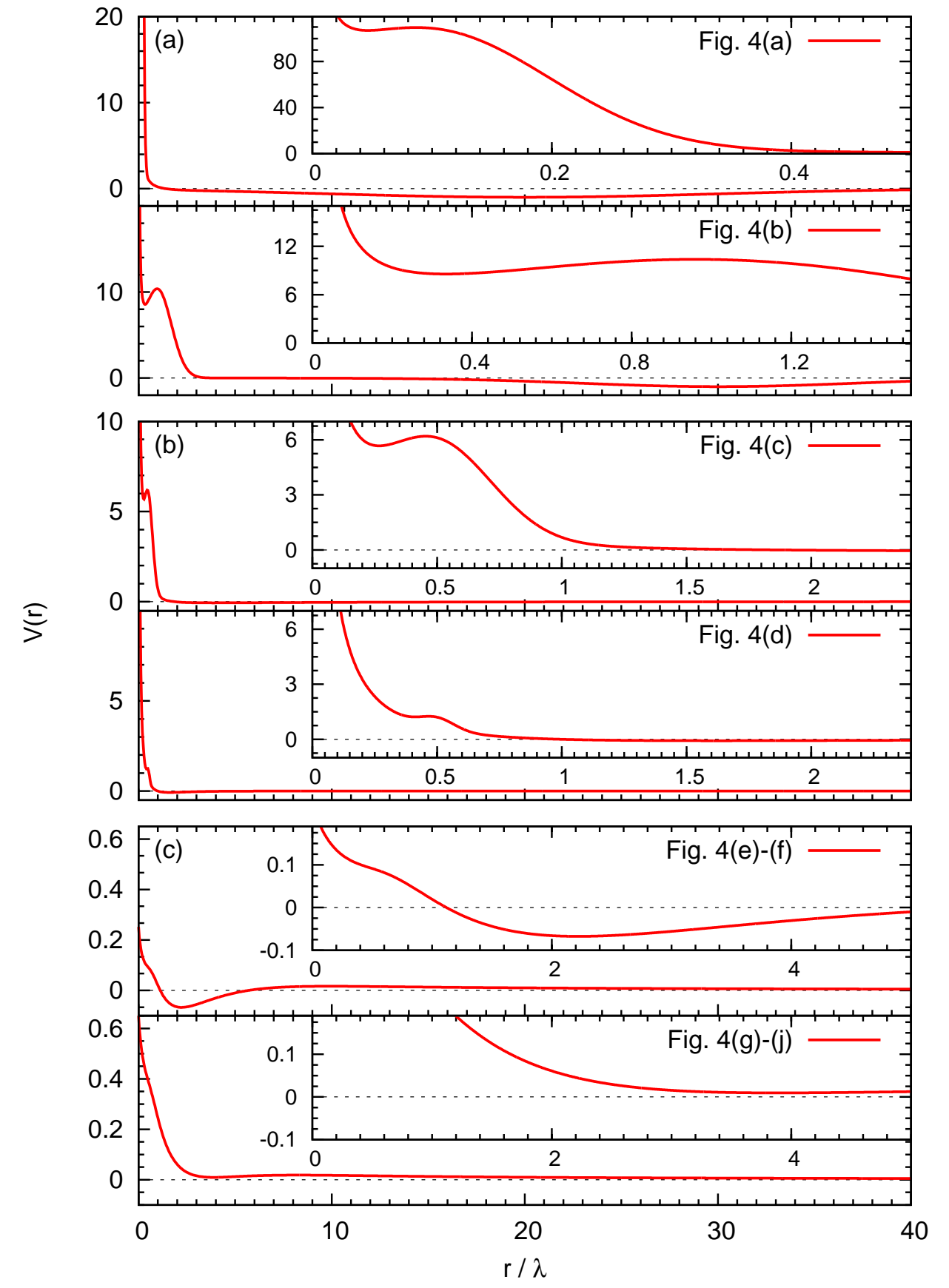

Figure 3: Inter-vortex pair potentials used in this study. Panels (a), (b), and (c) each illustrate a different pair potential, with the top and bottom of each panel representing a different set of parameters (see Appendix B for details). The insets are close up views of the potentials for small inter-particle separation. The legend indicates the corresponding panels in Figure 4.

inter-cluster distance inside a supercluster, with the subsequent peaks describing the distance between clusters in different superclusters.

A more physically accurate potential is achieved by smoothing out the steps in the 


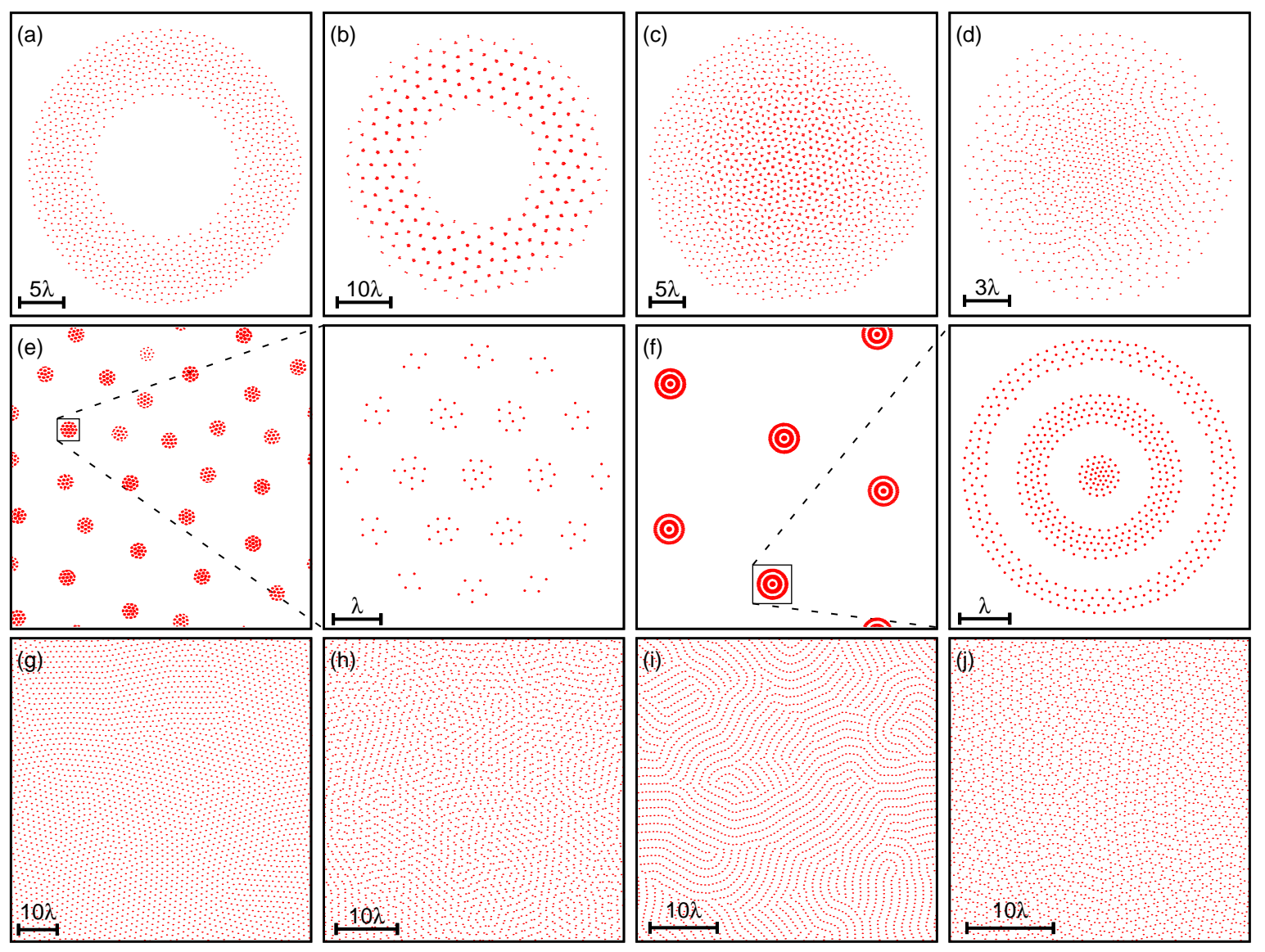

Figure 4: Snapshot of the final vortex configurations corresponding to the potentials in (a) top panel of Figure 3(a) with $N_{v}=1000$ and density $\rho=0.044$, (b) bottom panel of Figure 3(a) with $N_{v}=1000$ and density $\rho=0.025$, (c) top panel of Figure 3(b) with $N_{v}=2000$ and $\rho=0.20$, (d) bottom panel of Figure 3(b) with $N_{v}=1000$ and $\rho=0.40$, top panel of Figure 3(c) with $N_{v}=3000$ and densities (e) $\rho=0.25$ and (f) $\rho=1.00$, and the bottom panel of Figure 3(c) with $N_{v}=3000$ and densities (g) $\rho=0.50$, (h) $\rho=1.25$, (i) $\rho=1.50$, and (j) $\rho=2.50$. For panels (a), (b), (c), and (d), the long-range attraction causes all of the particles to form a single object and we only show a close up view. The unlabeled panels are close-up views of panels (e) and (f), focusing on a single supercluster and ring, respectively. The final vortex configurations in panels (a)-(d) and panels (e-j) are from MC and LD simulations, respectively, with simulation details discussed in the Appendices.

previous potential [see Figure 1(b)]. In Figure 2(b), we show the final MC configuration for the smooth potential, which is a supercluster of higher radial symmetry. The RDF is plotted in Figure 2(d) and has nearly identical features, although the higher symmetry of the ground state results in smoother peaks. The conclusions that follow are (i) multiple repulsive length scales result in a formation of hierarchical structures and (ii) the precise form of the potential is of a lesser importance in this example: the crucial aspect is the existence of several length scales in the interaction. 


\section{General Layered Systems}

To classify possible hierarchical structures we need to consider what sorts of vortex structures exist in systems where there exist competing interactions that are dominant at different length scales (see Figure 3). In the context of Figure 1, such systems can be realized by adding layers of type- 1 material or alternating layers of clean and dirty material, while repulsive scales are tunable by controlling e.g. layer thicknesses.

In Figure 3(a), we show two potentials that both feature a strong repulsive core surrounded by an area of attraction. Outside the attractive shell, there is a repulsive region and an attractive long-range coupling. At very low vortex densities, the final MC configurations for both of these potentials are given in Figure 4(a) and Figure 4(b), respectively. In the first case, the particles form a cluster due to their attractive interaction, the repulsive scale however gives this cluster a ring shape. In the second, the combination of attractive and repulsive scales induces clustering inside the ring (a clustered ring).

Next, we consider two potentials where the long-range attraction is extremely weak and we vary the potential at intermediate length scales [see Figure 3(b)]. The final MC configurations for these potentials at densities $\rho=0.2$ and $\rho=0.8$ are shown in Figures. 4(c) and 4(d). In the first case, the particles form a single large supercluster with one critical difference from the case shown on Figure 2: the size of the constituent small clusters is modulated by the distance to the center of the cluster, going from a maximum of 4 vortices per cluster in the center to a shell of single vortices at the edge. In the second case, when the interaction in the intermediate region is modified, the local phase of the cluster varies with distance from the center: namely as one goes from the center of the cluster to the edge one encounters regions corresponding to vortex lattice, vortex stripes, and vortex voids phases. Here the long range attractive interaction makes the vortex density gradually increase towards the center of the cluster leading to a sequence of phases which optimize the interaction associated with repulsive short-range scales.

The third pair of potentials [see Figure 3(c)] we examine feature a moderate repulsive core surrounded by an attractive well and have a long-range repulsive interaction. When the well is strong, the final vortex configurations from LD simulations [39] are circular superclusters at a density $\rho=0.25$ or concentric rings at a density of $\rho=1$ [illustrated in Figures. 4(e) and 4(f)]. Again the short-range structure is determined by repulsive length scales. However, when the attractive well is weakened significantly more regular repulsion-dominated vortex phases appear at higher densities [see Figures. 4(g)-(j)]: a triangular lattice, a pair vortex lattice, stripes, and voids, which is consistent with a dominance of short-range two-scale repulsive interactions.

To better understand the structure of these phases, let us examine the RDF for each

phase, which are shown in Figure 5 (note that the ordering of the panels matches the ordering of Figure 4). For the ring phase of Figure 4(a), $g(r)$ has three pronounced peaks indicating the nearest-neighbor distance, next-nearest-neighbor distance, etc. inside the ring. In between the peaks, $g(r)$ remains finite because the vortices do not form an even 
Heirarchical structure formation in layered superconducting systems...

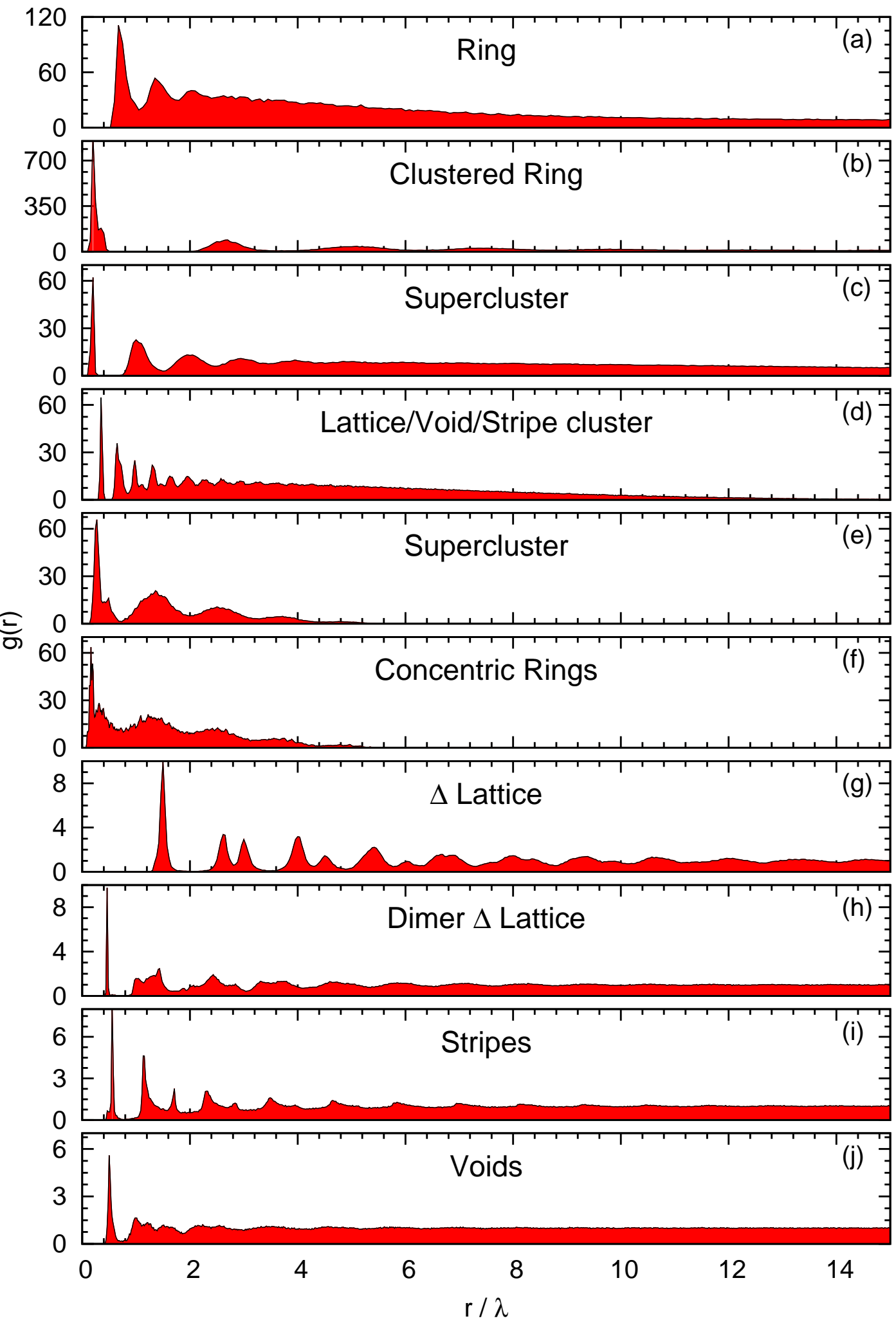

Figure 5: Radial distribution function $g(r)$ corresponding to the phases illustrated in Figure 4. 
lattice inside the ring. For the clustered ring phase [see Figure 4(b)], the first peak is very pronounced and narrow, indicative of the particles in each cluster being roughly equidistant. The second peak characterizes nearest-neighbor distance between clusters, and the subsequent peaks the distance between next-nearest-neighboring clusters, etc. The long distances between the peaks indicate that the clusters are small compared to their separation.

The modulated supercluster of Figure 4(c) likewise has a sharp, narrow peak in $g(r)$ representing the nearly equidistant particle separation inside each cluster. As with the clustered ring, the ensuing peaks represent the distances between neighboring clusters and the broadness of the peaks is due to the variability of cluster size throughout the structure. For the stripe/void-rich phase [see Figure 4(d)], we observe several prominent peaks in $g(r)$ that are largely consistent with the RDF for a triangular lattice, with broadening of the peaks due to the mixing of phases.

The superclusters shown in Figure 4(e) possess short range periodicity inside each cluster, resulting in two narrow peaks in $g(r)$ which are so close together that the second peak appears as a shoulder. The successive peaks illustrate the distance between clusters and are broadened because of the finite size of each cluster. Because the clusters are widely separated, there are additional broad peaks in $g(r)$ that occur for large $r$ and describe the supercluster separation. The RDF of the concentric ring phase [pictured in Figure 4(f)] is remarkably similar to the $g(r)$ for the supercluster phase [Figure 4(e)]. Unlike the supercluster phase, $g(r)$ remains finite for $r$ smaller than the diameter of the outer ring due to the particles spreading out evenly throughout each ring.

Finally, we discuss the radial distribution functions of more conventionally ordered phases. The lattice phase [see Figure $5(\mathrm{~g})$ ] possesses much more long-range order than

all other phases considered, with peaks at $r=a, \sqrt{3} a, 2 a, \sqrt{7} a, 3 a, \ldots$, where $a$ is the nearest-neighbor distance for a triangular lattice. The dimer lattice phase [see Figure 5(h)] has a clearly defined peak describing the dimer separation. Because the pairs prefer to line up end-to-end, the peaks that would describe the triangular lattice are broadened significantly and the long-range order cannot be observed in $g(r)$. The stripe phase [see Figure 5(i)] has several regularly spaced peaks coinciding perfectly with the separation of particles along each stripe. Here, broadening occurs due to both bending of the stripes and the presence of other stripes. The void phase [see Figure 5(j)] only has short-range periodicity, as evidenced by the pronounced features for small $r$.

\section{Summary}

In this paper, we presented MC and LD simulations of vortex states for a model of vortex-vortex interactions in general layered superconductor-insulator-superconducting structures, made of different superconducting layers. The vortices are subject to interactions with multiple length scales. We have shown that these layered systems have an unusual magnetic response: vortex supercluster structures, which can consist of clusters of clusters, rings, clusters in a ring, or have coexistence of stripes, voids, 
and lattice phases. This can provide an experimental tool to deduce information about vortex interactions from observation of their ordering in real experiments. Besides that it indicates that one can use layered superconducting structures for the realization and study of rich and unique pattern forming systems. More generally, our results indicate that systems with additional characteristic length scales may exhibit more complicated hierarchies in structure formation. In such systems, increasing the number of repulsive or attractive length scales should result in multiple hierarchy orderings, yielding "fractal crystal" phases in the limit of a large number of length scales.

In further studies we plan to quantitatively determine the multi-scale intervortex interactions by solving coupled Ginzburg-Landau and Maxwell equations for superconductor-insulator multi-layers.

\section{Acknowledgments}

We thank M. Touminen and C. Santangelo for discussions. This work was supported by NSF Award No. DMR-0955902 (C.N.V., Q.W. and E.B.) by Knut and Alice Wallenberg Foundation through the Royal Swedish Academy of Sciences (E.B.), Swedish Research Council (E.B. AND K.S.). The computations were partially performed on resources provided by the Swedish National Infrastructure for Computing (SNIC) at National Supercomputer Center at Linkoping, Sweden.

\section{Appendix A. Methods Summary}

In this paper we have utilized both $\mathrm{MC}$ and $\mathrm{LD}$ simulations to obtain the vortex structure formations at $T=0$.

Monte Carlo. The vortex structure formations are obtained using the Metropolis MC algorithm, where at each $\mathrm{MC}$ iteration a randomly chosen vortex is displaced a distance $(0, d]$ chosen at random, and MC moves are accepted or rejected according to the Metropolis MC scheme. The potential energy is calculated with a sharp cut off of the interaction potential at half the box length. The presented results are snapshots at zero temperature after at least $10^{3}$ sweeps from an random initial configuration, where temperature was incrementally lowered to zero. The step length $d$ was typically set to the box length.

Langevin Dynamics. The dynamics of a vortex are described by the overdamped Langevin equation of motion

$$
\eta \frac{d \mathbf{r}_{i}}{d t}=\mathbf{F}_{i}^{v v}+\mathbf{F}_{i}^{T},
$$

where $\eta$ is the damping constant, $\mathbf{r}_{i}$ is the position of the $i$-th vortex, $\mathbf{F}_{i}^{v v}=-\nabla V_{i j}$ is the inter-vortex force, and $\mathbf{F}_{i}^{T}$ is the stochastic thermal force. The simulation is performed with periodic boundary conditions and the vortex-vortex interaction is cut 
off smoothly $[34,40]$. The equation of motion is integrated by an Euler scheme with a reduced time step of $\Delta t=0.005$. We measure length in units of $\lambda=200 \AA$. In all cases, the initial configuration of the vortices is randomly distributed, and the temperature is incrementally lowered to zero, with at least $2 \times 10^{5}$ integration steps at each temperature. For 3000 vortices, each time step takes $\sim 2.2 \mathrm{~s}$ on a single AMD processor with a clock speed of $2.2 \mathrm{GHz}$.

Radial Distribution Function. The phases are analyzed by means of the radial distribution function (RDF) [38]

$$
g(r)=\frac{1}{2 \pi r \Delta r \rho N} \sum_{i=1}^{N} n_{i}(r, \Delta r)
$$

where $n_{i}(r, \Delta r)$ is the number of particles in the shell surrounding the $i$ th particle with radius $r$ and thickness $\Delta r$. For small distances, $g(r) \rightarrow 0$. Meanwhile, for large distances the radial distribution function must approach unity, i.e. $g(r) \rightarrow 1$ as $r \rightarrow \infty$.

\section{Appendix B. Potentials}

In order to understand the competition between interactions acting at different length scales, we first consider a core-softened hard-sphere model with multiple shoulders

$$
V(r)=\left\{\begin{array}{cc}
\infty & r<\sigma_{h} \\
\varepsilon_{1} & \sigma_{h}<r<\sigma_{1} \\
\varepsilon_{2} & \sigma_{1}<r<\sigma_{2} \\
0 & r>\sigma_{2}
\end{array},\right.
$$

where $\sigma_{h}$ is the hard-core radius, $\sigma_{1}$ and $\sigma_{2}$ are the shoulder radii, and $\varepsilon_{1}$ and $\varepsilon_{2}$ are the shoulder heights (see Fig. 1(a) for a sketch of a vortex in this potential). In this work, we performed MC simulations with $N_{v}=2000$ vortices, various densities and parameters $\sigma_{h}=0.25, \sigma_{1}=1.75, \sigma_{2}=10, \varepsilon_{1}=2.25$, and $\varepsilon_{2}=0.8$. The ground state of this pair potential for a wide range of densities is the supercluster phase illustrated in Fig. 2(a).

The key feature responsible for different structure formations is the number of repulsive/attractive length scales and their relative values. The precise shape of the potential is less important. A more physical realization of this potential can be obtained by smoothing out the steps [see Fig. 1(b)]. This smoothed potential can be modeled by

$$
V(r)=\left(K_{0}(r)+e^{-(r-1)^{2}}+1\right) e^{-1 /(10-r)},
$$

where $K_{0}$ is a modified Bessel function of the second kind, $r$ is the separation between particles, and $V(r)=0$ for $r \geq 10$. In all cases studied, we found the ground state to be similar to the ground state of Equation (B.1).

As discussed in the main text, general layered systems have competing interactions that are not necessarily repulsive. Attractive parts can arise from core-core interactions 
in layers of type-1 material. Figure 3 of the main text illustrates several scenarios considered in this study. The potential form of Fig. 3(a) is modeled by

$$
\frac{V(r)}{V_{0}}=\frac{e^{-r / \lambda}}{r / \lambda}-c_{1} e^{-\alpha_{1}\left(r /-\beta_{1}\right)^{2}}+c_{2} e^{-\alpha_{2}\left(r / a-\beta_{2}\right)^{2}},
$$

where $c_{1}, \alpha_{1}, \beta_{1}, c_{2}, \alpha_{2}$, and $\beta_{2}$ are coefficients, $V_{0}$ sets the unit of energy, and $\lambda$ is the characteristic length scale of magnetic field localization. In what follows, we set $\lambda=1$ as the unit of length. The values of these coefficients corresponding to the top and bottom panels of Fig. 3(a) are $c_{1}=1.0, \alpha_{1}=0.005, \beta_{1}=20, c_{2}=100, \alpha_{2}=50, \beta_{2}=0.1$ and $c_{1}=1, \alpha_{1}=0.01, \beta_{1}=30, c_{2}=10, \alpha_{2}=1.0, \beta_{2}=1.0$, respectively.

The potential form corresponding to Fig. $3(\mathrm{~b})$ is very similar to Equation (B.3),

$$
\frac{V(r)}{V_{0}}=\frac{e^{-r / \lambda}}{r / \lambda}-c_{1} e^{-\alpha_{1}\left(r /-\beta_{1}\right)}+c_{2} e^{-\alpha_{2}\left(r /-\beta_{2}\right)^{2}} .
$$

Here the coefficients for the top and bottom panels are $c_{1}=0.1, \alpha_{1}=0.1, \beta_{2}=0.0$, $c_{2}=5.0, \alpha_{2}=10, \beta_{2}=0.5$ and $c_{1}=1.0, \alpha_{1}=1.0, \beta_{2}=0.0, c_{2}=0.6, \alpha_{2}=100$, $\beta_{2}=0.5$, respectively.

The potential for Fig. 3(c) is

$$
\frac{V(r)}{V_{0}}=e^{-r / \lambda}-c_{2} e^{-r / \xi}+c_{3} \frac{\lambda\{\tanh [\alpha(r-\beta)]+1\}}{r+\delta},
$$

It models a short-range exponential repulsion, an intermediate-ranged exponential attraction, and a long-range power law term resulting from stray fields. For the third term, the onset of the long-range force is mediated by the parameters $a, b$, and $\delta$, which ensure that the dominant short-range force is the first term. For both the top and bottom panels, we consider $c_{3}=0.1, \alpha=2.5, \beta=0.5$, and $\delta=0.1$. The remaining parameters are $c_{2}=0.9$ and $\xi=1.80$ for the top panel and $c_{2}=0.5$ and $\xi=1.85$ for the bottom panel.

\section{References}

[1] Olson Reichhardt C J, Reichhardt C and Bishop A R 2010 Phys. Rev. E 82(4) 041502

[2] Olson Reichhardt C J, Reichhardt C and Bishop A R 2011 Phys. Rev. E 83(4) 041501

[3] Spivak B and Kivelson S A 2004 Phys. Rev. B 70(15) 155114

[4] Smørgrav E, Smiseth J, Babaev E and Sudbø A 2005 Phys. Rev. Lett. 94(9) 096401

[5] Parameswaran S A, Kivelson S A, Rezayi E H, Simon S H, Sondhi S L and Spivak B Z 2012 Phys. Rev. B 85241307

[6] Ravenhall D G, Pethick C J and Wilson J R 1983 Phys. Rev. Lett. 50(26) 2066-2069

[7] Malescio G and Pellicane G 2003 Nature Materials 2 97-100

[8] Glaser M A, Grason G M, Kamien R D, Komrlj A, Santangelo C D and Ziherl P 2007 Europhys. Lett. 7846004 
[9] Liu Y H, Chew L Y and Yu M Y 2008 Phys. Rev. E 78(6) 066405

[10] Abrikosov A A 1957 Sov. Phys. JETP 51174

[11] Blatter G, Feigel'man M V, Geshkenbein V B, Larkin A I and Vinokur V M 1994 Rev. Mod. Phys. 66(4) 1125-1388

[12] Babaev E and Speight M 2005 Phys. Rev. B 72(18) 180502

[13] Moshchalkov V, Menghini M, Nishio T, Chen Q H, Silhanek A V, Dao V H, Chibotaru L F, Zhigadlo N D and Karpinski J 2009 Phys. Rev. Lett. 102(11) 117001

[14] Nishio T, Dao V H, Chen Q, Chibotaru L F, Kadowaki K and Moshchalkov V V 2010 Phys. Rev. B 81(2) 020506

[15] Dolocan V O, Veauvy C, Servant F, Lejay P, Hasselbach K, Liu Y and Mailly D 2005 Phys. Rev. Lett. 95(9) 097004

[16] Björnsson P G, Maeno Y, Huber M E and Moler K A 2005 Phys. Rev. B 72(1) 012504

[17] Curran P J, Khotkevych V V, Bending S J, Gibbs A S, Lee S L and Mackenzie A P 2011 Phys. Rev. B 84(10) 104507

[18] Geurts R, Milošević M V and Peeters F M 2010 Phys. Rev. B 81(21) 214514

[19] Carlström J, Babaev E and Speight M 2011 Phys. Rev. B 83(17) 174509

[20] Carlström J, Garaud J and Babaev E 2011 Phys. Rev. B 84(13) 134515

[21] Lin S Z and Hu X 2011 Phys. Rev. B 84(21) 214505

[22] Silaev M and Babaev E 2011 Phys. Rev. B 84(9) 094515

[23] Silaev M and Babaev E 2012 Phys. Rev. B 85(13) 134514

[24] Dao V H, Chibotaru L F, Nishio T and Moshchalkov V V 2011 Phys. Rev. B 83(2) 020503

[25] Garaud J, Agterberg D F and Babaev E 2012 Phys. Rev. B 86(6) 060513

[26] Drocco J A, Reichhardt C J O, Reichhardt C and Bishop A R 2013 J. Phys.: Condens. Matt. 25345703

[27] Gutierrez J, Raes B, Silhanek A V, Li L J, Zhigadlo N D, Karpinski J, Tempere J and Moshchalkov V V 2012 Phys. Rev. B 85(9) 094511

[28] Stojanović V M, Vincent Liu W and Kim Y B 2008 Annals of Physics 323 989-1023

[29] Alford M G and Good G 2008 Phys. Rev. B 78(2) 024510

[30] Babaev E, Carlström J, Garaud J, Silaev M and Speight J 2012 Physica C 479 $2-14$

[31] Babaev E 2008 Phys. Rev. B 77(5) 054512

[32] Pearl J 1964 Appl. Phys. Lett. 565

[33] Landau D and Binder K 2005 A Guide to Monte Carlo Simulations in Statistical Physics (Cambridge: Cambridge University Press)

[34] Fangohr H, Cox S J and de Groot P A J 2001 Phys. Rev. B 64(6) 064505 
[35] Malescio G and Pellicane G 2004 Phys. Rev. E 70(2) 021202

[36] Camp P J 2003 Phys. Rev. E 68(6) 061506

[37] Camp P J 2005 Phys. Rev. E 71(3) 031507

[38] Hansen J P and McDonald I R 1986 Theory of Simple Liquids (New York: Dover)

[39] Although long-range ordering of the superclusters and concentric rings is not present in the final LD configurations shown Figs. 4(e) and 4(f), simulations of $N_{v}=1000$ and $N_{v}=2000$ vortices exhibit similar long-lived metastable configurations which ultimately order in a lattice.

[40] Fangohr H, Price A R, Cox S J, de Groot P A, Daniell G J and Thomas K S 2000 J. Comp. Phys. $162372-384$ 